\title{
Doppelbesprechung
}

\section{Die digitalisierte Gesellschaft}

Christoph Kucklick, Die granulare Gesellschaft. Wie das Digitale unsere Wirklichkeit auflöst. Berlin: Ullstein 2015, 268 S., gb., 18,00€

Deborah Lupton, Digital Sociology. London \& New York: Routledge 2015, 230 S., kt., $37,50 €$

Besprochen von PD Dr. phil. Axel Philipps: Wissenschaftlicher Mitarbeiter am Institut für

Soziologie der Leibniz Universität Hannover, E-Mail: a.philipps@ish.uni-hannover.de

DOI 10.1515/srsr-2015-0075

Schlüsselwörter: Digitale Soziologie, Digitalisierung, Big Data, digitale Werkzeuge

Die Reduktion der Vielfalt an Zeichen und Symbolen auf wenige, wie beim Binärcode auf 0 und 1, hat die Voraussetzungen geschaffen, dass Maschinen verschiedenartige Informationen verarbeiten können. Automatische Handlungsanweisungen (Algorithmen) erlauben in der Folge den Austausch von Informationen zwischen technischen Geräten. Und vor allem die Nutzung dieser Möglichkeiten produziert enorme Datenmengen, die man bisher in erster Linie aus der Quantenphysik kannte (Mainzer, 2014). Während Unternehmen und Regierungen frühzeitig begannen, diese riesigen Mengen an Daten für eigene Zwecke zu nutzen, beschäftigt das Phänomen unter dem Begriff Big Data die geistes- und sozialwissenschaftlichen Debatten erst in jüngster Zeit (etwa Geiselberger, 2013; Reichert, 2014). In diese Reihe von Abwägungen über die Chancen und Risiken von Big Data bringen die beiden hier vorzustellenden Bücher eine deutlich zu erkennende soziologische Perspektive ein. Sie setzen sich beide mit der Frage auseinander, wie der Gebrauch von digitalen Technologien die Menschen, ihr Leben und die Gesellschaft verändert. Im Mittelpunkt stehen vor allem digitale Computer als Kombination aus physischen Elementen (Hardware) und automatisch operierenden Handlungsanweisungen (Software) und wie diese eingesetzt werden, mit menschlichen Aktivitäten verschmelzen und welche Konsequenzen daraus erwachsen.

Beide Bücher nähern sich dem Thema jedoch auf ganz unterschiedliche Weise an. So geht Christoph Kucklick in seinem Buch „Die granulare Gesellschaft“ von der These einer durch Digitalisierung „feinaufgelösten Gesellschaft“ (Kucklick: 10) aus, um im ersten Teil des Buches anhand verschiedenartiger Bei- 
spiele, Szenarien und Anekdoten der Charakteristik dieser detailgenau abbildbaren Gesellschaft nachzuspüren. Er liefert darin Variationen des bekannten Bildes vom gläsernen Kunden, dessen aufgezeichnetes Einkaufsverhalten persönlich zugeschnittene Angebote und Kaufanreize ermöglicht. Kucklick verwendet dazu eine einfache, eingängige und teilweise dramatisierende Sprache, um schließlich im zweiten Buchteil die daraus erwachsenen Folgen zu thematisieren oder vielmehr seine Vision vom „granularen Menschen“ zu entwickeln.

Deborah Lupton steigt ebenfalls mit der These von der digitalisierten Gesellschaft ein. Auch sie gibt anschauliche Beispiele dafür, wie stark digitale Technologien bereits unser Leben und die Gesellschaft durchdringen und diskutiert, welche Konsequenzen daraus folgen. Sie beschränkt sich jedoch nicht auf eine Innenansicht der digitalisierten Gesellschaft. Sie versucht ebenfalls die Entwicklungen aus einer soziologischen Perspektive zu erklären. Zugleich entwickelt sie keine bestimmte These, sondern gibt vielmehr einen Überblick zum Thema. In einer typisch wissenschaftlichen Form führt sie in Theorien, Methoden und verschiedene Themenfelder zur digitalisierten Gesellschaft ein.

Kucklick arbeitet ebenfalls mit wissenschaftlichen Belegen, hat diese jedoch ans Ende verbannt, um ungestört von Literaturverweisen oder Fußnoten das Bild einer „Neuen Auflösung“ (Kucklick: 10) zu entwerfen. Demnach erfährt das Wissen über die Menschen eine bisher ungekannte Detailgenauigkeit. Es beruht nicht mehr auf Durchschnittswerten aggregierter Daten, sondern die Menge und Art der Daten erlaubt, jeden einzelnen Menschen $\mathrm{zu}$ rekonstruieren. Diese Entwicklung hat nach Kucklick drei Revolutionen zur Folge, welche er im ersten Teil des Buches beschreibt. Durch die erste Revolution werden die Menschen „radikal vereinzelt, singularisiert“ (Kucklick: 11). Mit den digitalen Technologien entstehen zweitens neue Anforderungen an Wissen und Know-how. Vor allem bringen sie neue Formen der Arbeitsteilung mit sich. So heißt es: „Computer übernehmen die gut definierbaren Jobs mit algorithmisch zerlegbaren Tätigkeiten, Menschen übernehmen alles, was vage, schwer zu definieren und komplex ist“ (Kucklick: 83). Die dritte Revolution zeichnet sich darin aus, dass das Verhalten der Menschen mittels der digitalen Technologien nicht mehr diszipliniert, sondern durch Anreize kontrolliert wird. Der zweite Teil des Buches thematisiert zwei Konsequenzen aus den Entwicklungen. Zum einen greifen die bisherigen bewährten Problemlösungen und Expertisen nicht mehr. Wie sind daher die digitalen Technologien und ihre Möglichkeiten $\mathrm{zu}$ kanalisieren? Wie lassen sich beispielsweise die automatischen Algorithmen der Vermessung offen legen, „ohne dadurch die berechtigten Interessen von Firmen und Staaten an Geheimhaltung zu ignorieren“ (Kucklick: 169)? Zum anderen sieht Kucklick das Problem, dass die Grenze zwischen Mensch und Maschine verwischt. Um Eindeutigkeit wieder herzustellen, braucht es demnach eine neue Selbstbeschreibung, deren 
Kontur sich für ihn in der Gestalt des granularen Menschen abzeichnet: „Er wird spielend experimentieren, um die Maschinen $\mathrm{zu}$ begreifen. Er wird voller Empathie sein, um die Differenzen zu anderen zu überbrücken. Und er wird launisch sein und unberechenbar, um die Mechanismen der gesellschaftlichen Kontrolle nach Kräften zu unterlaufen“ (Kucklick: 233).

Kucklick geht es in erster Linie darum, die Facetten der granularen Gesellschaft vorzustellen. Er bietet dazu viele anschauliche Beispiele, wie Informationen zu Waren werden und soziale Netzwerke die Arbeit am eigenen Selbst bestärken. Wie er aber selbst schreibt, will er sich nicht mit dem Neoliberalismus als „vermeintlichem Bösewicht“ (Kucklick: 17) auseinandersetzen. So erfahren wir beispielsweise etwas über die „Quantified Self“-Bewegung, deren Mitglieder mit Hilfe digitaler Technologien Daten über sich und ihre Körper sammeln und diese Daten auswerten. Kucklick belässt die Erklärung des Verhaltens bei einer „Bewegung aus Einzelnen, die etwas nur über sich in Erfahrung bringen“ (Kucklick: 49) wollen. Woher kommt aber diese Motivation, „sich zu verändern, um fitter zu werden“ (Kucklick: 50)? Er liefert dazu keine Interpretation des Verhaltens als Verinnerlichung neoliberaler Anforderungen, an sich Selbst zu arbeiten, sich selbst zum Projekt zu machen. Ganz im Gegensatz zu Lupton in ihrem Buch (siehe Lupton: 181).

Grundsätzlich stellte sich mir die Frage, wie wahrscheinlich es ist, mit dem „granularen Menschen“ zu rechnen. Die Darstellungen Kucklicks auf den Seiten $88 \mathrm{ff}$. zum spielerischen Experimentieren und Unberechenbaren erinnern an das Bild des „Werdens“ bei Deleuze und Guattari (1992). Es geht eben nicht darum, vom bestehenden Wissen weiterzudenken, sondern Überraschendes durch beständiges Zerlegen, Verbinden und Modifizieren hervorzubringen. So ist auch der Gedanke nicht neu, dass Web als Rhizom zu betrachten, als „ein azentrisches, nicht hierarchisches und asignifikantes System ohne General“ (Deleuze / Guattari, 1992: 35) und ohne organisiertes Gedächtnis. Kritische Betrachtungen des rhizomatischen Webs legen jedoch nahe (Hess, 2008; Tredinnick, 2013), dass die Mehrheit der Nutzerinnen und Nutzer das Web auf stabilisierte und hierarchisch strukturierte Weisen gebraucht. Der experimentierfreudige und unberechenbare Mensch bleibt demnach eher die Ausnahme.

Im Buch „Digital Sociology“ verfolgt Lupton ein anderes Ziel. Ausgehend von der Beobachtung, wie stark digitale Technologien bereits unseren Alltag prägen, möchte sie die Soziologie an den Gegenstand heranführen und zeigt in Grundzügen die Themen einer digitalen Soziologie auf. Sie geht dazu von der These eines zunehmenden Gebrauchs digitaler Technologien im Alltag aus. Im Mittelpunkt stehen die enormen Datenbestände (Big Data), welche sowohl durch die Verknüpfung und den Austausch zwischen den digitalen Werkzeugen als auch durch das Hochladen und Teilen von Inhalten über soziale Medien entstehen. Die 
Menschen werden dadurch „digital data subjects“ (Lupton: 3), die viel über ihre Präferenzen, Vorstellungen und Verhaltensweisen preisgeben. Das Buch möchte daher einen Einblick geben in die sozialen, kulturellen und politischen Dimensionen der digitalen Gesellschaft und anregen, das Phänomen kritisch zu betrachten.

Nach Lupton forscht die „digitale Soziologie“ allgemein zum Gebrauch digitaler Technologien und ist selbstreflexiv, wenn es darum geht, solche Technologien in der Soziologie zu nutzen (Lupton: 15). Im Buch unterscheidet sie dazu vier Forschungsfelder: 1) wie gebrauchen Soziologinnen und Soziologen digitale Technologien (professional digital practice), 2) wie nutzen die Menschen allgemein solche Technologien (analyses of digital technology use), 3) wie lassen sich die großen Datenmengen methodisch erschließen (digital data analysis) und 4) wie kann eine soziologisch informierte Kritik digitaler Technologien aussehen (critical digital sociology).

Die thematische Struktur des Buches weicht damit leicht von den identifizierten Forschungsfeldern ab, welche DiMaggio und andere (2001) für die Soziologie noch im Jahr 2001 ausmachten. Sie sahen einen Forschungsbedarf zu Fragen der 1) sozialen Ungleichheit (the „digital divide“), 2) der politischen Partizipation, 3) der kulturellen Mitgestaltung und Vielfalt sowie 4) zum Verständnis von Community und sozialem Kapital und 5) von webbasierten Organisationen und anderen wirtschaftlichen Unternehmen. Lupton stellt dazu nicht nur aktuelle und ausgewählte Forschungen vor, sie zeigt auch das gewachsene Selbstbewusstsein der Soziologie, sich kritisch mit dem Gebrauch digitaler Technologien auseinanderzusetzen.

Ein eigenes Kapitel liefert einen Überblick $\mathrm{zu}$ verschiedenen theoretischen Ansätzen und Konzepten. Darin schreibt sie über die Informationsgesellschaft (Castell) und wie die digital zugänglichen Informationen zum Wirtschaftsfaktor werden, oder wie die Actor-Network-Theorie digitale Technologien als nichtmenschliche Akteure zu menschlichen Akteuren in Beziehung setzt. Über den Neoliberalismus kommt sie auf das Konzept der Prosumption zu sprechen, wonach Nutzerinnen und Nutzer digitaler Werkzeuge eigene Inhalte bereitstellen und die anderer konsumieren. Durch die Prosumption entstehen wiederum Informationen, welche im Kapitalismus einen Wert für wirtschaftliche Zwecke generieren. Schließlich nehmen Theorien zur Überwachung (theories of veillance) und Hybridität einen größeren Stellenwert ein. Die einen thematisieren die neuen Dimensionen der Überwachung (etwa Umfang, Speichermöglichkeiten und Vordringen ins Private) und die anderen, wie sich die klare Abgrenzung von Mensch und Maschine auflöst.

Ein weiteres Kapitel stellt aktuelle Forschungsergebnisse zur digitalen Ungleichheit, zu digitalen Überwachungspolitiken, politischem Aktivismus, sozia- 
len Bewegungen und digital vermittelter Selbstwahrnehmung vor. In Ergänzung zu DiMaggio et al. (2001) gehen zudem zwei Kapitel näher auf verfügbare methodische Instrumentarien ein, welche den Soziologinnen und Soziologen zur Verfügung stehen (Suchmaschinen, Visualisierungswerkzeuge, Tracking-Software und andere). Der Einblick beschränkt sich jedoch auf die Empfehlung eines eher spielerischen Umgangs mit den verfügbaren digitalen Werkzeugen. Sie sollten genutzt werden, „to think in unexpected and inventive ways“ (Lupton: 54). Das Buch liefert aber keinen Überblick zu Verfahren wie Data-Mining oder ähnlichem. Während solche Möglichkeiten im Abschnitt zu „native digital data“ (im Web erzeugte Daten) schlicht als Häufigkeitszählungen (Lupton: 55) abgetan werden, erfährt das Thema Big Data eine größere Aufmerksamkeit. Lupton beleuchtet kritisch zum einen die wachsende Bedeutung solcher Daten für kommerzielle, staatliche und persönliche Zwecke und zum anderen die noch fehlenden Kenntnisse der Soziologinnen und Soziologen darüber, wie „native digital data“ entstehen und was sie bedeuten. Ein weiteres Kapitel betrachtet dazu eingehend den Ursprung und die Verbreitung der Metapher Big Data und welche Versprechungen als auch welcher wirtschaftliche und staatliche Gebrauch daraus erwachsen.

Quer zu den eher allgemein gehaltenen Überblickskapiteln steht ein Abschnitt zur Nutzung digitaler Technologien unter Akademikerinnen und Akademikern. Darin stellt Lupton eine eigene Studie vor, in der sie das Nutzungsverhalten sowie die damit verbundenen Vor- und Nachteile für die akademische Welt untersucht. So ermöglicht das Web einerseits den ausgegrenzten und peripheren Gruppen, sich zu artikulieren (Lupton: 83). Andererseits sind diese Gruppen dadurch auch stärker der Diskriminierung ausgesetzt (Lupton: 86).

Den roten Faden im Buch bilden die digitalen Technologien und Werkzeuge („digital devices“ bzw. „digital technologies“). Demnach geht es weniger um eine Darstellung der „Digital Sociology“ als vielmehr um die „sociology of digital technologies/devices“. Folglich löst das Buch den eigenen Anspruch der Autorin nicht ein, das Digitale in seinem gesamten Spektrum abzubilden und auch die soziologische Forschung dazu vorzustellen (Lupton: 5). Völlig ausgeblendet bleibt beispielsweise die wissens- und kultursoziologische Perspektive auf die Digitalisierung und welche Konsequenzen sich aus der Übersetzung von Zeichen und Symbolen in eine maschinenlesbare Binärstruktur für die Konstruktion, Wandelbarkeit und Vielfältigkeit von Wissen ergeben. Wie schreiben sich das Wissen und die Erfahrung der Menschen in die Konstruktion von Algorithmen für Übersetzungen und Austausch ein? Wie strukturiert die Digitalisierung umgekehrt unsere Wissensbestände und Formen der Weltaneignung? Das Thema wird kurz angerissen (Lupton: 24ff.), es fehlen aber Einblicke in Forschungsfelder wie das Konzept mediatisierter Welten (Krotz / Hepp, 2012), zu den wechselseitigen 
Identitätskonstruktionen im Web (Burnett / Marshall, 2003) oder zu der durch Digitalisierung hervorgebrachten Visual Culture (Mirzoeff, 2002).

Grundsätzlich habe ich zudem Bedenken, ob eine digitale Soziologie überhaupt in der Lage wäre, soziale Phänomene im Zusammenhang mit dem Digitalen umfassend $\mathrm{zu}$ verstehen und $\mathrm{zu}$ erklären. Es ist unmöglich, aus der Perspektive einer digitalen Soziologie die Forschungsfelder der gesamten Soziologie zu überblicken. Bei Lupton wird dies immer dann deutlich, wenn sie ihre Argumentation eng ans Digitale bindet und kaum weiterführende Forschung heranzieht. So findet sich im Buch ein Abschnitt zum Forschungsfeld digitaler Protest und soziale Bewegungen. Darin thematisiert sie Cyberprotest und andere Formen, lässt jedoch weitere Erklärungsmodelle aus der Bewegungsforschung (etwa Gelegenheitsstrukturen, Ressourcenmobilisierung, Frameanpassung) unberücksichtigt. Um solche Verkürzungen zu vermeiden, sollte sich die digitale Soziologie darauf konzentrieren, spezifische Theorien und Forschungen bereitzustellen, welche von den weiteren Forschungsfeldern in der Soziologie integriert werden können.

Des Weiteren vergibt Lupton die Chance, die Soziologie an digitale Werkzeuge heranzuführen. Sie stellt zwar Softwarewerkzeuge vor, jedoch in erster Linie Suchmaschinen und Softwarelösungen, die Ergebnisse der Suchmaschinen visualisieren. Eine weitere Beschäftigung scheint sich aus der kritischen Haltung zum Gebrauch webbasierter Metadaten zu verbieten. So erfahren wir kaum etwas darüber, wie mit Hilfe der verfügbaren Metadaten und Data-Mining-Verfahren soziologische Fragestellungen bearbeitet werden können. Dazu muss man nicht unbedingt die Annahme von Savage und Burrows (2007) von einer kommenden Krise der Soziologie teilen. Die Soziologie war zwar maßgeblich an der Entwicklung von Verfahren wie Umfragen und Paneluntersuchungen beteiligt, die auch ökonomisch und politisch relevant wurden. Ihre Zukunft hängt aber nicht allein davon ab, die Methoden zur Analyse digitaler Daten mit zu entwickeln. Sie kann jedoch davon profitieren, wenn sie mit digitalen Daten vertraut ist, sie zu analysieren und sie $\mathrm{zu}$ interpretieren weiß. Wer sich dafür interessiert und einen weiteren Zugang nutzen will, das Soziale zu erforschen, möge Bail (2014), Lemke und Wiedemann (2015), Wiedemann (2013) oder Mohr und Bogdanov (2013) konsultieren.

Insgesamt ist Kucklicks Buch mit der zugespitzten These einer Gesellschaft, welche detaillierte, permanent aktualisierte Daten über alle ihre Bewegungen und Handlungen verfügt, sehr lesenswert. Er stellt die dringende Frage danach, was eine solche Gesellschaft aus uns macht. Wobei offen bleibt, ob es je soweit kommt. Sein anregend geschriebenes und gut nachvollziehbares Gedankenmodell eignet sich gut, stärker über die Bedeutung digitaler Technologien im Alltag nachzudenken. 
Lupton richtet sich in ihrem Buch hingegen stärker an ein wissenschaftliches Publikum, welches auf der Suche nach einem soziologischen Überblick zur Bedeutung digitaler Technologien im alltäglichen Gebrauch ist. Hierzu gibt sie anschauliche Einblicke in die Möglichkeiten und das Ausmaß unternehmerischer und staatlich gesteuerter Überwachung und Kontrolle. Durch die vielen anschaulichen Beispiele und die leicht verständliche Sprache eignet sich das Buch für den Einstieg in die Thematik ebenso wie für einen Überblick zum Stand der internationalen Forschung.

\section{Literatur}

Bail, C. A. The cultural environment: measuring culture with big data. Theory and Society 2014, 43, 465-482.

Burnett, R.; Marshall, P. D. Web theory: An introduction; Routledge: London, 2003.

Deleuze, G.; Guattari, F. Tausend Plateaus: Kapitalismus und Schizophrenie; Merve: Berlin, 1992. DiMaggio, P.; Hargittai, E.; Neuman, W. R.; Robinson, J. P. Social implications of the Internet. Annual Review of Sociology 2001, 27, 307-336.

Geiselberger, H. Hrsg. Big Data: Das neue Versprechen der Allwissenheit; Suhrkamp: Frankfurt a. M., 2013.

Hess, A. Reconsidering the rhizome: A textual analysis of web search engines as gatekeepers of the internet. In Web Search; Spink, A.; Zimmer, M., Eds.; Springer: Berlin, Heidelberg, 2008; pp 35-50.

Krotz, F.; Hepp, A. Hrsg. Mediatisierte Welten: Forschungsfelder und Beschreibungsansätze; Springer VS: Wiesbaden, 2012.

Lemke, T.; Wiedemann, G. Hrsg. Text Mining in den Sozialwissenschaften. Grundlagen und Anwendungen zwischen qualitativer und quantitativer Diskursanalyse; Springer VS: Wiesbaden, 2015.

Mainzer, K. Die Berechnung der Welt: Von der Weltformel zu Big Data; Beck: München, 2014.

Mirzoeff, N. Ed. The visual culture reader. London: Routledge, 2002.

Mohr, J. W.; Bogdanov, P. Introduction - Topic models: What they are and why they matter. Poetics 2013, 41, 545-569.

Reichert, R. Hrsg. Big Data: Analysen zum digitalen Wandel von Wissen, Macht und Ökonomie; transcript: Bielefeld, 2014.

Savage, M.; Burrows, R. The coming crisis of empirical sociology. Sociology 2007, 41, 885-899.

Tredinnick, L. Each One of us was Several: Networks, Rhizomes and Web Organisms. Knowledge Organization 2013, 40, 414-421.

Wiedemann, Gregor (2013). Opening up to Big Data: Computer-Assisted Analysis of Textual Data in Social Sciences [54 paragraphs]. Forum Qualitative Sozialforschung / Forum: Qualitative Social Research, 14(2), Art. 13, http://nbn-resolving.de/urn:nbn:de:0114-fqs1302231. 